\title{
A NOTE ON CLASSICAI HILBERT INEQUALITY
}

\author{
GOU-SHENG YANG, SHYH-LIANG GAU
}

\begin{abstract}
In this note we establish some new generalizations of the classical inequality of Hilbert with non-conjugate parameters.
\end{abstract}

\section{Introduction}

Let $x_{m} \geq 0, x_{m} \not \equiv 0, y_{n} \geq 0, y_{n} \not \equiv 0, p>1, q>1, \frac{1}{p}+\frac{1}{p^{\prime}}=1, \frac{1}{q}+\frac{1}{q^{\prime}}=1$

The inequality:

$$
\sum_{m=1}^{\infty} \sum_{n=1}^{\infty} \frac{x_{m} y_{n}}{m+n}<\pi \csc \left(\frac{\pi}{p}\right)\left(\sum_{m=1}^{\infty} x_{m}^{p}\right)^{\frac{1}{p}}\left(\sum_{n=1}^{\infty} y_{n}{ }^{p^{\prime}}\right)^{\frac{1}{p^{\prime}}}
$$

is well-known generalization of the classical inequality of Hilbert, where the constant $\pi \csc \left(\frac{\pi}{p}\right)$ is best possible. (see for instance Th.315 from [2]).

The case when instead of $p, p^{\prime}$ we have non-conjugate parameters $p, q$ was also consider in [2].

If

$$
p>1, \quad q>1, \quad \frac{1}{p}+\frac{1}{q} \geq 1
$$

such that

$$
0<\lambda=2-\frac{1}{p}-\frac{1}{q}=\frac{1}{p^{\prime}}+\frac{1}{q^{\prime}} \leq 1
$$

then

$$
\sum_{m=1}^{\infty} \sum_{n=1}^{\infty} \frac{x_{m} y_{n}}{(m+n)^{\lambda}}<K(p, q, \lambda)\left(\sum_{m=1}^{\infty} x_{m}^{p}\right)^{\frac{1}{p}}\left(\sum_{n=1}^{\infty} y_{n}^{q}\right)^{\frac{1}{q}}
$$

Received June 22, 1994; revised September 15, 1994.

1991 Mathematics Subject Classificatio .. 26D15.

Key words and phrases. Inequality of Hilbert, Hölder inequality. 
where

$$
K(p, q, \lambda)=\left(\pi \csc \frac{\pi}{\lambda p^{\prime}}\right)^{\lambda}=\left(\pi \csc \frac{\pi}{\lambda q^{\prime}}\right)^{\lambda}[\operatorname{see} 1,4,5] .
$$

In [3] Mitrinović and Pečarić proved that, if

$$
\lambda>0, \quad \frac{1}{p}+\frac{1}{q}>\lambda \geq \frac{1}{p^{\prime}}+\frac{1}{q^{\prime}}
$$

then

$$
K(p, q, \lambda)=\left(\pi \csc \left(\frac{\pi}{2 \lambda}\left(\frac{1}{p}-\frac{1}{q}+\lambda\right)\right)\right)^{\lambda} .
$$

which follows from (1) and the following

Theorem $\mathbf{A}$. Let $a_{m n} \geq 0$ and suppose that for every $p>1$ and $x_{m} \geq 0, y_{n} \geq 0$ the follwoing inequality is valid

$$
\sum_{m=1}^{\infty} \sum_{n=1}^{\infty} a_{m n} x_{m} y_{n} \leq K(p)\left(\sum_{m=1}^{\infty} x_{m}^{p}\right)^{\frac{1}{p}}\left(\sum_{n=1}^{\infty} y_{n}^{p^{\prime}}\right)^{\frac{1}{p^{\prime}}}
$$

where $p^{\prime}, p$ are conjugate i.e. $\frac{1}{p}+\frac{1}{p^{\prime}}=1$.

If $p>1, q>1, \frac{1}{p}+\frac{1}{q} \geq 1$ and $\lambda>0, \frac{1}{p}+\frac{1}{q}>\lambda \geq \frac{1}{p^{\prime}}+\frac{1}{q^{\prime}}$, then

$$
\sum_{m=1}^{\infty} \sum_{n=1}^{\infty} a_{m n}^{\lambda} x_{m} y_{n} \leq K^{\lambda}\left(\left(\frac{1}{2 \lambda}\left(\frac{1}{p}-\frac{1}{q}+\lambda\right)\right)^{-1}\right)\left(\sum_{m=1}^{\infty} x_{m}^{p}\right)^{\frac{1}{p}}\left(\sum_{n=1}^{\infty} y_{n}^{q}\right)^{\frac{1}{q}}
$$

The following theorems are stated without proof in [3].

Theorem B. Let $k(x, y) \geq 0$ and suppose that for every $p>1$ and $f(x) \geq$ $0, g(y) \geq 0$ the following inequality is valid

$$
\iint k(x, y) f(x) g(y) d x d y \leq K(p)\left(\int f^{p}(x) d x\right)^{\frac{1}{p}}\left(\int g^{p^{\prime}}(y) d y\right)^{\frac{1}{p^{\prime}}} .
$$

If $p>1, \quad q>1, \quad \frac{1}{p}+\frac{1}{q} \geq 1$ and $\lambda>0, \quad \lambda=\frac{1}{p^{\prime}}+\frac{1}{q^{\prime}}$, then

$$
\iint k^{\lambda}(x, y) f(x) g(y) d x d y \leq K^{\lambda}\left(\lambda q^{\prime}\right)\left(\int f^{p}(x) d x\right)^{\frac{1}{p}}\left(\int g^{q}(y) d y\right)^{\frac{1}{q}} .
$$

Theorem $\mathbb{C}$. Let $a_{m n \ldots s} \geq 0$ and suppose that for every $p_{1}>1, p_{2}>1, \cdots$, $p_{r}>1$ s.t. $\sum_{i=1}^{r} p_{i}^{-1}=1$ and $x_{m} \geq 0, y_{n} \geq 0, \ldots, z_{s} \geq 0$ the following inequality is 
valid

$$
\begin{gathered}
\sum a_{m n \ldots s} x_{m} y_{n} \ldots z_{s} \leq \\
\qquad\left(p_{1}, p_{2}, \ldots, p_{r}\right)\left(\sum_{m=1}^{\infty} x_{m}{ }^{p_{1}}\right)^{\frac{1}{p_{1}}}\left(\sum_{n=1}^{\infty} y_{n}{ }^{p_{2}}\right)^{\frac{1}{p_{2}}} \\
\ldots\left(\sum_{s=1}^{\infty} z_{s}^{p_{r}}\right)^{\frac{1}{p_{r}}} .
\end{gathered}
$$

If $q_{i}>1, i=1,2, \ldots, r$ and $\lambda>0,(r-1) \lambda \geq \sum_{i=1}^{r} 1 / q_{i}^{\prime}, \sum_{j=1}^{r} 1 / q_{j}>\lambda, i=$ $1,2, \ldots, r$, then

$$
\begin{gathered}
\sum a_{m n \ldots s}^{\lambda} x_{m} y_{n} \cdots z_{s} \leq K^{\lambda}\left(\underline{p}_{1}, \underline{p}_{2}, \ldots, \underline{p}_{r}\right)\left(\sum_{m=1}^{\infty} x_{m}^{q_{1}}\right)^{\frac{1}{q_{1}}}\left(\sum_{n=1}^{\infty} y_{n}{ }^{q_{2}}\right)^{\frac{1}{q_{2}}} \\
\ldots\left(\sum_{s=1}^{\infty} z_{s}{ }^{q_{r}}\right)^{\frac{1}{q_{r}}}
\end{gathered}
$$

where $\frac{1}{\underline{p}_{i}}=\frac{1}{r \lambda}\left(\frac{r}{q_{i}}-\sum_{j=1}^{r} \frac{1}{q_{j}}+\lambda\right), i=1,2, \ldots, r$.

Theorem D. Let $k(x, y, \ldots, z) \geq 0$ and suppose that for every $p_{1}>1, p_{2}>$ $1, \cdots, p_{r}>1$ s.t. $\sum_{i=1}^{r} p_{i}^{-1}=1$ and $f(x) \geq 0, g(y) \geq 0, \ldots, h(z) \geq 0$ the following inequality is valid

$$
\begin{gathered}
\iint \cdots \int k(x, y, \ldots, z) f(x) g(y) \cdots h(z) d x d y \cdots d z \leq K\left(p_{1}, p_{2}, \ldots, p_{r}\right) \\
\left(\int f^{p_{1}}(x) d x\right)^{\frac{1}{p_{1}}} \cdot\left(\int g^{p_{2}}(y) d y\right)^{\frac{1}{p_{2}}} \cdots\left(\int h^{p_{r}}(z) d z\right)^{\frac{1}{p_{r}}} .
\end{gathered}
$$

If $q_{i}>1, \quad i=1,2, \ldots, r$ and $0<\lambda<1, \sum_{i=1}^{r} \frac{1}{q_{i}}>1,(r-1) \lambda=\sum_{i=1}^{r} \frac{1}{q_{i}^{\prime}}$, then

$$
\begin{gathered}
\iint \cdots \int k^{\lambda}(x, y, \ldots, z) f(x) g(y) \cdots h(z) d x d y \cdots d z \leq K^{\lambda}\left(\bar{p}_{1}, \bar{p}_{2}, \ldots, \bar{p}_{r}\right) \\
\left(\int f^{q_{1}}(x) d x\right)^{\frac{1}{q_{1}}}\left(\int g^{q_{2}}(y) d y\right)^{\frac{1}{q_{2}}} \cdots\left(\int h^{q_{r}}(z) d z\right)^{\frac{1}{q_{r}}},
\end{gathered}
$$

where $\bar{p}_{i}=\lambda\left(\lambda-1+\frac{1}{q_{i}}\right)^{-1}, i=1,2, \ldots, r$.

The aim of this note is to extended Theorem A and give proofs of Theorem B, Theorem $\mathrm{C}$ and Theorem $\mathrm{D}$ in general forms.

\section{Main results}

Theorem 1. Let $c>0, s \geq 0, t \geq 0$ s.t. $s+c \geq 1, t+c \geq 1$ and $f(m) \geq$ $0, g(n) \geq 0$ 
Let $a_{m n} \geq 0$ and suppose that for every $p>1$ and $x_{m} \geq 0, y_{n} \geq 0$ the following inequality is valid

$$
\sum_{m=1}^{\infty} \sum_{n=1}^{\infty} a_{m n} x_{m} y_{n} \leq K\left(\frac{p}{c}\right)\left(\sum_{m=1}^{\infty} x_{m}^{\frac{p}{c}}\right)^{\frac{c}{p}}\left(\sum_{n=1}^{\infty} y_{n} \frac{p^{\prime}}{c}\right)^{\frac{c}{p^{\prime}}} .
$$

If $p>1, q>1, \frac{1}{p}+\frac{1}{q} \geq 1$ and $\lambda>0, \frac{1}{p}+\frac{1}{q}>\lambda \geq \frac{1}{p^{\prime}}+\frac{1}{q^{\prime}}$, then

$$
\begin{gathered}
\sum_{m=1}^{\infty} \sum_{n=1}^{\infty} a_{m n}^{\lambda}\left(f^{s}(m) g^{t}(n)\right)^{k_{2}} x_{m} y_{n} \leq K^{\lambda}\left(\left(\frac{c}{2 \lambda}\left(\frac{1}{p}-\frac{1}{q}+\lambda\right)\right)^{-1}\right) \\
\left(\sum_{m=1}^{\infty} f(m)\right)^{s k_{2}}\left(\sum_{n=1}^{\infty} g(n)\right)^{t k_{2}}\left(\sum_{m=1}^{\infty} x_{m}^{\frac{p}{c}}\right)^{\frac{c}{p}}\left(\sum_{n=1}^{\infty} y_{n}^{\frac{q}{c}}\right)^{\frac{c}{q}},
\end{gathered}
$$

where $k_{2}=\frac{1}{2}\left(\frac{1}{p}+\frac{1}{q}-\lambda\right)$

Proof. We need the following

Lemma 1. (see [3]) Let $a_{m n} \geq 0, b_{m n} \geq 0, u_{1}>0, v_{1}>0, u_{2}>0, v_{2}>0$ and suppose that for every nonnegative $x_{m}, y_{n}$ the following inequalities are valid

$$
\begin{aligned}
& \sum_{m=1}^{\infty} \sum_{n=1}^{\infty} a_{m n} x_{m} y_{n} \leq K_{1}\left(\sum_{m=1}^{\infty} x_{m}^{\frac{1}{u_{1}}}\right)^{u_{1}}\left(\sum_{n=1}^{\infty} y_{n}^{\frac{1}{v_{1}}}\right)^{v_{1}}, \\
& \sum_{m=1}^{\infty} \sum_{n=1}^{\infty} b_{m n} x_{m} y_{n} \leq K_{2}\left(\sum_{m=1}^{\infty} x_{m}^{\frac{1}{n_{2}}}\right)^{u_{2}}\left(\sum_{n=1}^{\infty} y_{n}^{\frac{1}{v_{2}}}\right)^{v_{2}} .
\end{aligned}
$$

Futher, let $k_{1}>0, k_{2}>0, k_{1}+k_{2} \geq 1, u=k_{1} u_{1}+k_{2} u_{2}, v=k_{1} v_{1}+k_{2} v_{2}$.

Then

$$
\sum_{m=1}^{\infty} \sum_{n=1}^{\infty} a_{m n}^{k_{1}} b_{m n}^{k_{2}} x_{m} y_{n} \leq K_{1}{ }^{k_{1}} K_{2}{ }^{k_{2}}\left(\sum_{m=1}^{\infty} x_{m}{ }^{\frac{1}{u}}\right)^{u}\left(\sum_{n=1}^{\infty} y_{n}{ }^{\frac{1}{v}}\right)^{v} .
$$

Proof of Theorem 1 . If we set $b_{m n}=f^{s}(m) g^{t}(n), u_{1}=\frac{c}{p_{1}}, v_{1}=\frac{c}{p_{1}^{\prime}}, u_{2}=v_{2}=$ $c, k_{1}=\lambda, u=\frac{c}{p}, v=\frac{c}{q}$.

Since

$$
\left\{\begin{array}{l}
u=k_{1} u_{1}+k_{2} u_{2} \\
v=k_{1} v_{1}+k_{2} v_{2}
\end{array}\right.
$$

we have

$$
\left\{\begin{array}{l}
\frac{1}{p}=\lambda \frac{1}{p_{1}}+k_{2} \\
\frac{1}{q}=\lambda \frac{1}{p_{1}}+k_{2}
\end{array}\right.
$$


So that $k_{2}=\frac{1}{2}\left(\frac{1}{p}+\frac{1}{q}-\lambda\right)$ and $\frac{1}{p_{1}}=\frac{1}{2 \lambda}\left(\frac{1}{p}-\frac{1}{q}+\lambda\right)$.

The conditions of Lemma are fulfield, since we have:

$k_{2}>0$ and $k_{1}+k_{2}=\frac{1}{2}\left(\frac{1}{p}+\frac{1}{q}+\lambda\right) \geq \frac{1}{2}\left(\frac{1}{p}+\frac{1}{q}+\frac{1}{p^{\prime}}+\frac{1}{q^{\prime}}\right)=1$.

Now to check $p_{1}>1$ where $p_{1}=2 \lambda / \frac{1}{p}-\frac{1}{q}+\lambda$, we observe

$\langle i\rangle \frac{1}{p}-\frac{1}{q}+\lambda \geq \frac{1}{p}-\frac{1}{q}+\frac{1}{p^{\prime}}+\frac{1}{q^{\prime}}=1-\frac{1}{q}+\frac{1}{q^{\prime}}=\frac{2}{q^{\prime}}>0$

and $\langle i i\rangle 2 \lambda-\left(\frac{1}{p}-\frac{1}{q}+\lambda\right)=\lambda-\frac{1}{p}+\frac{1}{q} \geq \frac{1}{p^{\prime}}+\frac{1}{q^{\prime}}-\frac{1}{p}+\frac{1}{q}=1-\frac{1}{p}+\frac{1}{p^{\prime}}=\frac{2}{p^{\prime}}>0$.

It follows from $\langle i\rangle$ and $\langle i i\rangle$ that $p_{1}>1$. Now we have:

$$
\begin{aligned}
\sum_{m=1}^{\infty} \sum_{n=1}^{\infty} a_{m n} x_{m} y_{n} & \leq K\left(\frac{p_{1}}{c}\right)\left(\sum_{m=1}^{\infty} x_{m}^{\frac{p_{1}}{c}}\right)^{\frac{c}{p_{1}}}\left(\sum_{n=1}^{\infty} y_{n}^{\frac{p_{1}^{\prime}}{c}}\right)^{\frac{c}{p_{1}^{\prime}}} \\
& =K_{1}\left(\sum_{m=1}^{\infty} x_{m}^{\frac{1}{u_{1}}}\right)^{u_{1}}\left(\sum_{n=1}^{\infty} y_{n}^{\frac{1}{v_{1}}}\right)^{v_{1}}
\end{aligned}
$$

where $K_{1}=K\left(\frac{p_{1}}{c}\right)$.

Futhermore, Since $s+c \geq 1, t+c \geq 1$, we have (see [1], p29)

$$
\begin{aligned}
& \sum_{m=1}^{\infty} \sum_{n=1}^{\infty} f^{s}(m) g^{t}(n) x_{m} y_{n} \\
= & \sum_{m=1}^{\infty} \sum_{n=1}^{\infty} f^{s}(m) g^{t}(n)\left(x_{m}^{\frac{1}{c}}\right)^{c}\left(y_{n}^{\frac{1}{c}}\right)^{c} \\
\leq & \left(\sum_{m=1}^{\infty} f^{s}(m)\left(x_{m}^{\frac{1}{c}}\right)^{c}\right)\left(\sum_{n=1}^{\infty} g^{t}(n)\left(y_{n}^{\frac{1}{c}}\right)^{c}\right) \\
\leq & \left.\left(\sum_{m=1}^{\infty} f(m)\right)^{s}\left(\sum_{n=1}^{\infty} x_{m}^{\frac{1}{c}}\right)^{c}\left(\sum_{n=1}^{\infty} g(n)\right)^{t}\left(\sum_{n=1}^{\infty} y_{n}\right)^{\frac{1}{c}}\right)^{c} \\
= & K_{2}\left(\sum_{m=1}^{v_{2}} x_{m} \frac{1}{u_{2}}\right)^{v_{2}}\left(\sum_{n=1}^{\infty} y_{n}^{\frac{1}{v_{2}}}\right)^{,}
\end{aligned}
$$

where $K_{2}=\left(\sum_{m=1}^{\infty} f(m)\right)^{s}\left(\sum_{n=1}^{\infty} g(n)\right)^{t}$

It follows from Lemma 1 that

$$
\begin{gathered}
\sum_{m=1}^{\infty} \sum_{n=1}^{\infty} a_{m n}^{\lambda}\left(f^{s}(m) g^{t}(n)\right)^{k_{2}} x_{m} y_{n} \leq K^{\lambda}\left(\left(\frac{c}{2 \lambda}\left(\frac{1}{p}-\frac{1}{q}+\lambda\right)\right)^{-1}\right) \\
\left(\sum_{m=1}^{\infty} f(m)\right)^{s k_{2}}\left(\sum_{n=1}^{\infty} g(n)\right)^{t k_{2}}\left(\sum_{m=1}^{\infty} x_{m}^{\frac{p}{c}}\right)^{\frac{c}{p}}\left(\sum_{n=1}^{\infty} y_{n}^{\frac{q}{c}}\right)^{\frac{c}{q}},
\end{gathered}
$$

whrer $k_{2}=\frac{1}{2}\left(\frac{1}{p}+\frac{1}{q}-\lambda\right)$. This completes the proof. 
Theorem 2. Let $c \in(0,1], s \geq 0, t \geq 0$ s.t. $s+c=1, t+c=1$ and $w(x), r(y)$ are nonnegative integrable functions.

Let $k(x, y) \geq 0$ and suppose that for every $p>1$ and $f(x) \geq 0, g(y) \geq 0$ the following inequality is valid

$$
\iint k(x, y) f(x) g(y) d x d y \leq K\left(\frac{p}{c}\right)\left(\int f^{\frac{p}{c}}(x) d x\right)^{\frac{c}{p}}\left(\int g^{\frac{p^{\prime}}{c}}(y) d y\right)^{\frac{c}{p^{\prime}}} .
$$

If $p>1, q>1, \frac{1}{p}+\frac{1}{q} \geq 1$ and $\lambda>0, \lambda=\frac{1}{p^{\prime}}+\frac{1}{q^{\prime}}$, then

$$
\begin{gathered}
\iint k^{\lambda}(x, y)\left(w^{s}(x) r^{t}(y)\right)^{1-\lambda} f(x) g(y) d x d y \leq K^{\lambda}\left(\frac{\lambda q^{\prime}}{c}\right)\left(\int w(x) d x\right)^{s(1-\lambda)} \\
\left(\int r(y) d y\right)^{t(1-\lambda)}\left(\int f^{\frac{p}{c}}(x) d x\right)^{\frac{c}{p}}\left(\int g^{\frac{q}{c}}(y) d y\right)^{\frac{c}{q}}
\end{gathered}
$$

Proof. First, we need the following:

Lemma 2. Let $k(x, y) \geq 0, m(x, y) \geq 0, u_{1}>0, v_{1}>0, u_{2}>0, v_{2}>0$ and suppose that for every nonnegative $f(x), g(y)$ the following inequalities are valid

$$
\begin{gathered}
\iint k(x, y) f(x) g(y) d x d y \leq K_{1}\left(\int f(x)^{\frac{1}{u_{1}}} d x\right)^{u_{1}}\left(\int g(y)^{\frac{1}{v_{1}}} d y\right)^{v_{1}} \\
\iint m(x, y) f(x) g(y) d x d y \leq K_{2}\left(\int f(x)^{\frac{1}{u_{2}}} d x\right)^{u_{2}}\left(\int g(y)^{\frac{1}{v_{2}}} d y\right)^{v_{2}} .
\end{gathered}
$$

Futher, let $k_{1}>0, k_{2}>0, k_{1}+k_{2}=1, u=k_{1} u_{1}+k_{2} u_{2}, v=k_{1} v_{1}+k_{2} v_{2}$. Then

$$
\iint k^{k_{1}}(x, y) m^{k_{2}}(x, y) f(x) g(y) d x d y \leq K_{1}^{k_{1}} K_{2}^{k_{2}}\left(\int f(x)^{\frac{1}{u}} d x\right)^{u}\left(\int g(y)^{\frac{1}{v}} d y\right)^{v} \text {. }
$$

Proof of Lemma 2. By Hölder inequality, since $k_{1}+k_{2}=1$, it follows

$$
\begin{aligned}
& \iint k^{k_{1}}(x, y) m^{k_{2}}(x, y) f(x) g(y) d x d y \\
= & \iint k^{k_{1}}(x, y) m^{k_{2}}(x, y) f^{\frac{k_{1} u_{1}+k_{2} u_{2}}{u}}(x) g^{\frac{k_{1} v_{1}+k_{2} v_{2}}{v}}(y) d x d y \\
= & \iint\left(k(x, y) f^{\frac{u_{1}}{u}}(x) g^{\frac{v_{1}}{v}}(y)\right)^{k_{1}}\left(m(x, y) f^{\frac{u_{2}}{u}}(x) g^{\frac{v_{2}}{v}}(y)\right)^{k_{2}} d x d y \\
\leq & \left(\iint k(x, y) f^{\frac{u_{1}}{u}}(x) g^{\frac{v_{1}}{v}}(x) d x d y\right)^{k_{1}}\left(\iint m(x, y) f^{\frac{u_{2}}{n}}(x) g^{\frac{v_{2}}{v}}(y) d x d y\right)^{k_{2}} .
\end{aligned}
$$


CLASSICAL HILBERT INEQUALITY

87

As consequences of (2) and (3) we have

$$
\begin{aligned}
& \iint k(x, y) f^{\frac{u_{1}}{u}}(x) g^{\frac{v_{1}}{v}}(y) d x d y \leq K_{1}\left(\int f(x)^{\frac{1}{u}} d x\right)^{u_{1}}\left(\int g(y)^{\frac{1}{v}} d y\right)^{v_{1}} \\
& \iint m(x, y) f^{\frac{u_{2}}{u}}(x) g^{\frac{v_{2}}{v}}(y) d x d y \leq K_{2}\left(\int f(x)^{\frac{1}{u}} d x\right)^{u_{2}}\left(\int g(y)^{\frac{1}{v}} d y\right)^{v_{2}} .
\end{aligned}
$$

Substituting these inequalities into (4) we obtain:

$$
\begin{aligned}
& \iint k^{k_{1}}(x, y) m^{k_{2}}(x, y) f(x) g(y) d x d y \\
\leq & \left(K_{1}\left(\int f(x)^{\frac{1}{u}} d x\right)^{u_{1}}\left(\int g(y)^{\frac{1}{v}} d y\right)^{v_{1}}\right)^{k_{1}} \\
& \left(K_{2}\left(\int f(x)^{\frac{1}{u}} d x\right)^{u_{2}}\left(\int g(y)^{\frac{1}{v}} d y\right)^{v_{2}}\right)^{k_{2}} \\
= & K_{1}^{k_{1}} K_{2}^{k_{2}}\left(\int f(x)^{\frac{1}{u}} d x\right)^{k_{1} u_{1}+k_{2} u_{2}}\left(\int g(y)^{\frac{1}{v}} d y\right)^{k_{1} v_{1}+k_{2} v_{2}} \\
= & K_{1}^{k_{1}} K_{2}^{k_{2}}\left(\int f(x)^{\frac{1}{u}} d x\right)^{u}\left(\int g(y)^{\frac{1}{v}} d y\right)^{v} .
\end{aligned}
$$

Proof of Theorem 2. If we set: $m(x, y)=w^{s}(x) r^{t}(y), u_{1}=\frac{c}{p_{1}}, v_{1}=\frac{c}{p_{1}^{\prime}}$, $u_{2}=v_{2}=c, k_{1}=\lambda, u=\frac{c}{p}, v=\frac{c}{q}$, Since

$$
\left\{\begin{array}{l}
u=k_{1} u_{1}+k_{2} u_{2} \\
v=k_{1} v_{1}+k_{2} v_{2}
\end{array}\right.
$$

we have

$$
\left\{\begin{array}{l}
\frac{1}{p}=\lambda \frac{1}{p_{1}}+k_{2} \\
\frac{1}{q}=\lambda \frac{1}{p_{1}{ }^{\prime}}+k_{2}
\end{array} .\right.
$$

So that $k_{2}=1-\lambda$ and $p_{1}=\lambda\left(\frac{1}{p}-k_{2}\right)^{-1}$

$$
\begin{aligned}
& =\lambda\left(\frac{1}{p}-1+\lambda\right)^{-1} \\
& =\lambda\left(\frac{1}{p}-1+\frac{1}{p^{\prime}}+\frac{1}{q^{\prime}}\right)^{-1} \\
& =\lambda\left(-\frac{1}{p^{\prime}}+\frac{1}{p^{\prime}}+\frac{1}{q^{\prime}}\right)^{-1} \\
& =\lambda q^{\prime}=\left(\frac{1}{p^{\prime}}+\frac{1}{q^{\prime}}\right) q^{\prime}=\frac{q^{\prime}}{p^{\prime}}+1>1 .
\end{aligned}
$$


Now we have:

$$
\begin{aligned}
\iint k(x, y) f(x) g(y) d x d y & \leq K\left(\frac{p_{1}}{c}\right)\left(\int f^{\frac{p_{1}}{c}}(x) d x\right)^{\frac{c}{p_{1}}}\left(\int g^{\frac{p_{1}^{\prime}}{c}}(y) d y\right)^{\frac{c}{p_{1}{ }^{\prime}}} \\
& =K_{1}\left(\int f(x)^{\frac{1}{u_{1}}} d x\right)^{u_{1}}\left(\int g(y)^{\frac{1}{v_{1}}} d y\right)^{v_{1}}
\end{aligned}
$$

where $K_{1}=K\left(\frac{p_{1}}{c}\right)$.

Since $s+c=1, t+c=1$, it follows from Hölder inequality that:

$$
\begin{aligned}
& \iint w^{s}(x) r^{t}(y) f(x) g(y) d x d y \\
= & \iint w^{s}(x) r^{t}(y)\left(f^{\frac{1}{c}}(x)\right)^{c}\left(g^{\frac{1}{c}}(y)\right)^{c} d x d y \\
\leq & \left(\int w^{s}(x)\left(f^{\frac{1}{c}}(x)\right)^{c} d x\right)\left(\int r^{t}(y)\left(g^{\frac{1}{c}}(y)\right)^{c} d y\right) \\
\leq & \left(\int w(x) d x\right)^{s}\left(\int f^{\frac{1}{c}}(x) d x\right)^{c}\left(\int r(y) d y\right)^{t}\left(\int g^{\frac{1}{c}}(y) d y\right)^{c} \\
= & K_{2}\left(\int f(x)^{\frac{1}{u_{2}}} d x\right)^{u_{2}}\left(\int g(y)^{\frac{1}{v_{2}}} d y\right)^{v_{2}},
\end{aligned}
$$

where $K_{2}=\left(\int w(x) d x\right)^{s}\left(\int r(y) d y\right)^{t}$.

Applying Lemma 2 to obtain

$$
\begin{gathered}
\iint k^{\lambda}(x, y)\left(w^{s}(x) r^{t}(y)\right)^{1-\lambda} f(x) g(y) d x d y \leq K^{\lambda}\left(\frac{\lambda q^{\prime}}{c}\right)\left(\int w(x) d x\right)^{s(1-\lambda)} \\
\left(\int r(y) d y\right)^{t(1-\lambda)}\left(\int f^{\frac{p}{c}}(x) d x\right)^{\frac{c}{p}}\left(\int g^{\frac{q}{c}}(y) d y\right)^{\frac{c}{q}}
\end{gathered}
$$

This completes the proof of Theorem 2.

The followings are the multidimensional generlization of Theorem 1 and Theorem 2 .

Note. In theorem $\mathrm{C}$ and $\mathrm{D}$ implicitly is given that $\underline{p}_{i} \geq 1$. Hence, we can set, instead of $\sum_{i=1}^{r} 1 / q_{i}>\lambda$, the condition $\lambda+r / q_{i}>\sum_{i=1}^{r} 1 / q_{i}>\lambda, i=1,2, \ldots, r$, in Theorem $\mathrm{C}$, and instead of $\sum_{i=1}^{r} 1 / q_{i}>1$, the condition

$$
1+\frac{r-1}{q_{i}}>\sum_{i=1}^{r} 1 / q_{i}>1
$$

for $i=1,2, \ldots, r$ in the Theorem $D$.

Theorem 3. Let $c>0, \alpha \geq 0, \beta \geq 0, \ldots, \gamma \geq 0$ such that $\alpha+c \geq 1, \beta+c \geq 1$, $\ldots, \gamma+c \geq 1$ and $f(m), g(n), \ldots, h(s)$ are nonnegative. 
Let $a_{m n \cdots s} \geq 0$ and suppose that for every $p_{1}>1, p_{2}>1, \ldots, p_{r}>1$ s.t. $\sum_{i=1}^{r} p_{i}{ }^{-1}$ $=1$ and $x_{m} \geq 0, y_{n} \geq 0, \ldots, z_{s} \geq 0$ the follwoing inequality is valid

$$
\begin{aligned}
& \sum a_{m n \cdots s} x_{m} y_{n} \cdots z_{s} \\
\leq & K\left(\frac{p_{1}}{c}, \frac{p_{2}}{c}, \ldots, \frac{p_{r}}{c}\right)\left(\sum_{m=1}^{\infty} x_{m} \frac{p_{1}}{c}\right)^{\frac{c}{p_{1}}}\left(\sum_{n=1}^{\infty} y_{n}^{\frac{p_{2}}{c}}\right)^{\frac{c}{p_{2}}} \cdots\left(\sum_{s=1}^{\infty} z_{s}^{\frac{p_{r}}{c}}\right)^{\frac{c}{p_{r}}}
\end{aligned}
$$

If $q_{i}>1, i=1,2, \ldots, r$ and $\lambda>0,(r-1) \lambda \geq \sum_{i=1}^{r} 1 / q_{i}{ }^{\prime}, \lambda+\frac{r}{q_{i}}>\sum_{j=1}^{r} 1 / q_{j}>$ $\lambda, i=1,2, \ldots, r$, then

$$
\begin{aligned}
& \sum a_{m n \cdots s}^{\lambda}\left(f^{\alpha}(m) g^{\beta}(n) \cdots h^{\gamma}(s)\right)^{k_{2}} x_{m} y_{n} \cdots z_{s} \leq K^{\lambda}\left(\frac{\underline{p}}{c}, \frac{\underline{p}_{2}}{c}, \ldots, \frac{\underline{p}}{c}\right) \\
&\left(\sum_{m=1}^{\infty} f(m)\right)^{\alpha k_{2}}\left(\sum_{n=1}^{\infty} g(n)\right)^{\beta k_{2}} \cdots\left(\sum_{s=1}^{\infty} h(s)\right)^{\gamma k_{2}} \\
&\left.\left(\sum_{m=1}^{\infty} x_{m}{ }^{\frac{q_{1}}{c}}\right)^{\frac{c}{q_{1}}}\left(\sum_{n=1}^{\infty} y_{n}\right)^{\frac{q_{2}}{c}}\right)^{\frac{c}{q_{2}}}\left(\sum_{s=1}^{\infty} z_{a}^{\frac{q_{r}}{c}}\right)^{\frac{c}{q_{r}}}
\end{aligned}
$$

where $\frac{1}{\underline{p}_{i}}=\frac{1}{r \lambda}\left(\frac{r}{q_{i}}-\sum_{j=1}^{r} \frac{1}{q_{j}}+\lambda\right), i=1,2, \ldots, r, k_{2}=\left(\sum_{i=1}^{r} \frac{1}{q_{i}}-\lambda\right) / r$

Proof. We need the following :

Lemma 3. Let $a_{m n \cdots s} \geq 0, b_{m n \cdots s} \geq 0, u_{1}>0, v_{1}>0, \ldots, w_{1}>0, u_{2}>0, v_{2}>$ $0, \ldots, w_{2}>0$ and suppose that for every nonnegative $x_{m}, y_{n}, \ldots, z_{s}$ the following inequalities are valid

$$
\begin{aligned}
& \sum a_{m n \cdots s} x_{m} y_{n} \cdots z_{s} \leq K_{1}\left(\sum_{m=1}^{\infty} x_{m} \frac{1}{u_{1}}\right)^{u_{1}}\left(\sum_{n=1}^{\infty} y_{n} \frac{1}{v_{1}}\right)^{v_{1}} \cdots\left(\sum_{s=1}^{\infty} z_{s}^{\frac{1}{w_{1}}}\right)^{w_{1}} \\
& \sum b_{m n \cdots s} x_{m} y_{n} \cdots z_{s} \leq K_{2}\left(\sum_{m=1}^{\infty} x_{m} \frac{1}{u_{2}}\right)^{u_{2}}\left(\sum_{n=1}^{\infty} y_{n} \frac{1}{v_{2}}\right)^{v_{2}} \cdots\left(\sum_{s=1}^{\infty} z_{s}^{\frac{1}{w_{2}}}\right)^{w_{2}}
\end{aligned}
$$

Futher, let $k_{1}>0, k_{2}>0, k_{1}+k_{2} \geq 1, u=k_{1} u_{1}+k_{2} u_{2}, v=k_{1} v_{1}+k_{2} v_{2}, \ldots, w=$ $k_{1} w_{1}+k_{2} w_{2}$. Then

$$
\sum a_{m n \cdots s}^{k_{1} \ldots s} b_{m n \cdots s}^{k_{2}} x_{m} y_{n} \cdots z_{s} \leq K_{1}^{k_{1}} K_{2}^{k_{2}}\left(\sum_{m=1}^{\infty} x_{m}^{\frac{1}{u}}\right)^{u}\left(\sum_{n=1}^{\infty} y_{n}{ }^{\frac{1}{v}}\right)^{v} \cdots\left(\sum_{s=1}^{\infty} z_{s}^{\frac{1}{w}}\right)^{w} .
$$

Proof of Lemma 3. Using a generalization of Hölder inequality ( see [1], p29). 
Since $k_{1}+k_{2} \geq 1$, it follows

$$
\begin{aligned}
& \sum a_{m n \cdots s}^{k_{1}} b_{m n \cdots s}^{k_{2}} x_{m} y_{n} \cdots z_{s} \\
& =\sum\left(a_{m n \cdots s} x_{m}^{\frac{u_{1}}{u}} y_{m}{ }^{\frac{v_{1}}{v}} \cdots z_{s}^{\frac{w_{1}}{w}}\right)^{k_{1}}\left(b_{m n \cdots s} x_{m}{ }^{\frac{u_{2}}{v}} y_{n}{ }^{\frac{v_{2}}{v}} \cdots z_{s}^{\frac{w_{2}}{w}}\right)^{k_{2}} \\
& \leq\left(\sum a_{m n \cdots s} x_{m}^{\frac{u_{1}}{v}} y_{m}^{\frac{v_{1}}{v}} \cdots z_{s}^{\frac{w_{1}}{w}}\right)^{k_{1}}\left(\sum b_{m n \cdots s} x_{m}^{\frac{u_{2}}{u}} y_{n}^{\frac{w_{2}}{v}} \cdots z_{s}^{\frac{w_{2}}{w w}}\right)^{k_{2}} \text {. }
\end{aligned}
$$

As consequences of (5) and (6) we have:

$$
\begin{aligned}
& \sum a_{m n \cdots s} x_{m}{ }^{\frac{u_{1}}{k}} y_{m}{ }^{\frac{v_{1}}{v}} \cdots z_{s}^{\frac{w_{1}}{w}} \\
& \leq K_{1}\left(\sum_{m=1}^{\infty} x_{m}^{\frac{1}{u}}\right)^{u_{1}}\left(\sum_{n=1}^{\infty} y_{n}^{\frac{1}{v}}\right)^{v_{1}} \cdots\left(\sum_{s=1}^{\infty} z_{s}^{\frac{1}{w}}\right)^{w_{1}} \\
& \sum b_{m n \cdots s} x_{m}^{\frac{u_{2}}{v}} y_{n}^{\frac{v_{2}}{v}} \cdots z_{s}^{\frac{w_{2}}{w}} \\
& \leq K_{2}\left(\sum_{m=1}^{\infty} x_{m}^{\frac{1}{u}}\right)^{u_{2}}\left(\sum_{n=1}^{\infty} y_{n}^{\frac{1}{v}}\right)^{v_{2}} \cdots\left(\sum_{s=1}^{\infty} z_{s}^{\frac{1}{w}}\right)^{w_{2}} .
\end{aligned}
$$

Substituting these inequalities into (7) we obtain:

$$
\begin{aligned}
& \sum a_{m n \cdots s}^{k_{1}} b_{m n \cdots s}^{k_{2}} x_{m} y_{n} \cdots z_{s} \\
& \leq\left(K_{1}\left(\sum_{m=1}^{\infty} x_{m}{ }^{\frac{1}{u}}\right)^{u_{1}}\left(\sum_{n=1}^{\infty} y_{n} \frac{1}{v}\right)^{v_{1}} \cdots\left(\sum_{s=1}^{\infty} z_{s}^{\frac{1}{w}}\right)^{w_{1}}\right)^{k_{1}} \\
&\left(K_{2}\left(\sum_{m=1}^{\infty} x_{m}^{\frac{1}{u}}\right)^{u_{2}}\left(\sum_{n=1}^{\infty} y_{n}^{\frac{1}{v}}\right)^{v_{2}} \cdots\left(\sum_{s=1}^{\infty} z_{s}^{\frac{1}{w}}\right)^{w_{2}}\right)^{k_{2}} \\
&= K_{1}^{k_{1}} K_{2}^{k_{2}}\left(\sum_{m=1}^{\infty} x_{m}^{\frac{1}{u}}\right)^{k_{1} u_{1}+k_{2} u_{2}}\left(\sum_{n=1}^{\infty} y_{n}^{\frac{1}{v}}\right)^{k_{1} v_{1}+k_{2} v_{2}} \cdots\left(\sum_{s=1}^{\infty} z_{s}^{\frac{1}{w}}\right)^{k_{1} w_{1}+k_{2} w_{2}} \\
&= K_{1}^{k_{1}} K_{2}^{k_{2}}\left(\sum_{m=1}^{\infty} x_{m}^{\frac{1}{u}}\right)^{u}\left(\sum_{n=1}^{\infty} y_{n}^{\frac{1}{v}}\right)^{v} \cdots\left(\sum_{s=1}^{\infty} z_{s}^{\frac{1}{w}}\right)^{w} \cdot
\end{aligned}
$$

Proof of Theorem 3. If we set $b_{m n \cdots s}=f^{\alpha}(m) g^{\beta}(n) \cdots h^{\gamma}(s), u_{1}=\frac{c}{\underline{p}_{1}}, v_{1}=$ $\frac{c}{\underline{p}_{2}}, \ldots, w_{1}=\frac{c}{\underline{p}_{r}}$ s.t. $\sum_{i=1}^{r} \frac{1}{\underline{p}_{i}}=1, u_{2}=v_{2}=\cdots=w_{2}=c, k_{1}=\lambda, u=\frac{\underline{c}^{-}}{q_{1}}, v=$ $\frac{c}{q_{2}}, \ldots, w=\frac{c^{-}}{q_{r}}$. Since

$$
\left\{\begin{array}{l}
u=k_{1} u_{1}+k_{2} u_{2} \\
v=k_{1} v_{1}+k_{2} v_{2} \\
\vdots \\
w=k_{1} w_{1}+k_{2} w_{2}
\end{array}\right.
$$


we have

$$
\left\{\begin{array}{l}
\frac{1}{q_{1}}=\lambda \frac{1}{\underline{p}_{1}}+k_{2} \\
\frac{1}{q_{2}}=\lambda \frac{1}{\underline{p}_{1}}+k_{2} \\
\vdots \\
\frac{1}{q_{r}}=\lambda \frac{1}{\underline{p}_{r}}+k_{2} .
\end{array}\right.
$$

So that $k_{2}=\frac{\sum_{i=1}^{r} \frac{1}{q_{i}}-\lambda}{r} \geq 0$ and

$$
\begin{aligned}
\frac{1}{\underline{p}_{i}} & =\frac{1}{\lambda}\left(\frac{1}{q_{i}}-\frac{\sum_{j=1}^{r} \frac{1}{q_{j}}-\lambda}{r}\right) \\
& =\frac{1}{r \lambda}\left(\frac{r}{q_{i}}-\sum_{j=1}^{r} \frac{1}{q_{j}}+\lambda\right), \quad i=1,2, \ldots, r .
\end{aligned}
$$

The conditions of Lemma 3 are fulfield, since we have:

$$
\begin{aligned}
k_{2} \geq 0 \text { and } k_{1}+k_{2} & =\lambda+\frac{\sum_{i=1}^{r} \frac{1}{q_{i}}-\lambda}{r}=\frac{\sum_{i=1}^{r} \frac{1}{q_{i}}+\lambda(r-1)}{r} \\
& \geq \frac{\sum_{i=1}^{r} \frac{1}{q_{i}}+\sum_{i=1}^{r} \frac{1}{q_{i}{ }^{\prime}}}{r}=\frac{r}{r}=1 .
\end{aligned}
$$

Now to check $\underline{p}_{i}>1$, where $\underline{p}_{i}=r \lambda\left(\frac{r}{q_{i}}-\sum_{j=1}^{r} \frac{1}{q_{j}}+\lambda\right)^{-1}, i=1,2, \ldots, r$.

We observe $\langle i\rangle \frac{r}{q_{i}}-\sum_{j=1}^{r} \frac{1}{q_{j}}+\lambda>0$

$$
\text { and } \begin{aligned}
\langle i i\rangle r \lambda-\left(\frac{r}{q_{i}}-\sum_{j=1}^{r} \frac{1}{q_{j}}+\lambda\right) & =\sum_{j=1}^{r} \frac{1}{q_{j}}-\frac{r}{q_{i}}+\lambda(r-1) \\
& \geq \sum_{j=1}^{r} \frac{1}{q_{j}}-\frac{r}{q_{i}}+\sum_{j=1}^{r} \frac{1}{q_{j}{ }^{\prime}} \\
& =r-\frac{r}{q_{i}}=r\left(1-\frac{1}{q_{i}}\right)>0 \quad i=1,2, \ldots, r .
\end{aligned}
$$

It follows from $\langle i\rangle$ and $\langle i i\rangle$, that $\underline{p}_{i}>1, i=1,2, \ldots, r$. Now we have

$$
\begin{aligned}
\sum a_{m n \ldots s} x_{m} y_{n} \cdots z_{s} & \leq K\left(\frac{\underline{p}}{c}, \frac{\underline{\underline{p}}}{c}, \ldots, \frac{\underline{\underline{p}}}{c}\right)\left(\sum_{m=1}^{\infty} x_{m} \frac{\underline{p}_{1}}{c}\right)^{\frac{c}{\underline{p}_{1}}}\left(\sum_{n=1}^{\infty} y_{n} \frac{\underline{\underline{\underline{p}}}}{c}\right)^{\frac{c}{\underline{p}_{2}}} \\
& \ldots\left(\sum_{s=1}^{\infty} z_{s}^{\frac{\underline{p}_{r}}{c}}\right)^{\frac{c}{\underline{p}_{r}}} \\
& =K_{1}\left(\sum_{m=1}^{\infty} x_{m}^{\frac{1}{u_{1}}}\right)^{u_{1}}\left(\sum_{n=1}^{\infty} y_{n} \frac{1}{v_{1}}\right)^{v_{1}} \cdots\left(\sum_{s=1}^{\infty} z_{s}^{\frac{1}{w_{1}}}\right)^{w_{1}},
\end{aligned}
$$


where $K_{1}=K\left(\frac{\underline{\underline{p}}}{c}, \frac{\underline{p}_{2}}{c}, \ldots, \frac{\underline{p}_{r}}{c}\right)$.

Futhermore, Since $\alpha+c \geq 1, \beta+c \geq 1, \ldots, \gamma+c \geq 1$, we have (see [1], p29)

$$
\begin{aligned}
& \sum f^{\alpha}(m) g^{\beta}(n) \cdots h^{\gamma}(s) x_{m} y_{n} \cdots z_{s} \\
= & \sum f^{\alpha}(m) g^{\beta}(n) \cdots h^{\gamma}(s)\left(x_{m}{ }^{\frac{1}{c}}\right)^{c}\left(y_{n}{ }^{\frac{1}{c}}\right)^{c} \cdots\left(z_{s}{ }^{\frac{1}{c}}\right)^{c} \\
\leq & \left(\sum f^{\alpha}(m)\left(x_{m}{ }^{\frac{1}{c}}\right)^{c}\right)\left(\sum g^{\beta}(n)\left(y_{n}^{\frac{1}{c}}\right)^{c}\right) \cdots\left(\sum h^{\gamma}(s)\left(z_{s}^{\frac{1}{c}}\right)^{c}\right) \\
\leq & \left(\sum f(m)\right)^{\alpha}\left(\sum x_{m}^{\frac{1}{c}}\right)^{c}\left(\sum g(n)\right)^{\beta}\left(\sum y_{n}^{\frac{1}{c}}\right)^{c} \cdots\left(\sum h(s)\right)^{\gamma}\left(\sum z_{s}^{\frac{1}{c}}\right)^{c} \\
= & K_{2}\left(\sum_{m=1}^{\infty} x_{m}^{\frac{1}{u_{2}}}\right)^{u_{2}}\left(\sum_{n=1}^{\infty} y_{n}^{\frac{1}{v_{2}}}\right)^{v_{2}} \cdots\left(\sum_{s=1}^{\infty} z_{s}^{\frac{1}{w_{2}}}\right)^{w_{2}},
\end{aligned}
$$

where $K_{2}=\left(\sum f(m)\right)^{\alpha}\left(\sum g(n)\right)^{\beta} \cdots\left(\sum h(s)\right)^{\gamma}$.

It follows from Lemma 3 , that

$$
\begin{aligned}
& \sum a_{m n \cdots s}^{\lambda}\left(f^{\alpha}(m) g^{\beta}(n) \cdots h^{\gamma}(s)\right)^{k_{2}} x_{m} y_{n} \cdots z_{s} \\
\leq & K^{\lambda}\left(\frac{\underline{p}_{1}}{c}, \frac{\underline{p}_{2}}{c}, \ldots, \frac{\underline{p}_{r}}{c}\right)\left(\sum_{m=1}^{\infty} f(m)\right)^{\alpha k_{2}}\left(\sum_{n=1}^{\infty} g(n)\right)^{\beta k_{2}} \cdots\left(\sum_{s=1}^{\infty} h(s)\right)^{\gamma k_{2}} \\
& \left(\sum_{m=1}^{\infty} x_{m} \frac{\underline{q}_{1}}{c}\right)^{\frac{c}{q_{1}}}\left(\sum_{n=1}^{\infty} y_{n} \frac{\underline{q}_{2}}{c}\right)^{\frac{c}{q_{2}}} \cdots\left(\sum_{s=1}^{\infty} z_{s}^{\frac{q_{r}}{c}}\right)^{\frac{c}{q_{r}}}
\end{aligned}
$$

where $\frac{1}{\underline{p}_{i}}=\frac{1}{r \lambda}\left(\frac{r}{q_{i}}-\sum_{j=1}^{r} \frac{1}{q_{j}}+\lambda\right), i=1,2, \ldots, r, k_{2}=\left(\sum_{i=1}^{r} \frac{1}{q_{i}}-\lambda\right) / r$.

This completes the proof of Theorem 3 .

Theorem 4. Let $c \in(0,1], \alpha \geq 0, \beta \geq 0, \ldots, \gamma \geq 0$ such that $\alpha+c=1, \beta+c=$ $1, \ldots, \gamma+c=1$ and $w(x), r(y), \ldots, l(z)$ are nonnegative integrable functions.

Let $k(x, y, \ldots, z) \geq 0$ and suppose that for every $p_{1}>1, p_{2}>1, \ldots, p_{r}>1$ s.t. $\sum_{j=1}^{r} p_{i}^{-1}=1$ and $f(x) \geq 0, g(y) \geq 0, \ldots, h(z) \geq 0$ the following inequality is valid

$$
\begin{aligned}
& \iint \cdots \int k(x, y, \ldots, z) f(x) g(y) \cdots h(z) d x d y \cdots d z \\
\leq & K\left(\frac{p_{1}}{c}, \frac{p_{2}}{c}, \ldots, \frac{p_{r}}{c}\right)\left(\int f^{\frac{p_{1}}{c}}(x) d x\right)^{\frac{c}{p_{1}}}\left(\int g^{\frac{p_{2}}{c}}(y) d y\right)^{\frac{c}{p_{2}}} \cdots\left(\int h^{\frac{p_{r}}{c}}(z) d z\right)^{\frac{c}{p_{r}}} .
\end{aligned}
$$

If $q_{i}>1, i=1,2, \ldots, r$ and $0<\lambda<1,1+\frac{r-1}{q_{i}}>\sum_{j=1}^{r} \frac{1}{q_{j}}>1, i=1,2, \ldots, r$, 
$(r-1) \lambda=\sum_{j=1}^{r} \frac{1}{q_{j}^{\prime}}$, then

$$
\begin{aligned}
& \iint \cdots \int k^{\lambda}(x, y, \ldots, z)\left(w^{\alpha}(x) r^{\beta}(y) \cdots l^{r}(z)\right)^{1-\lambda} f(x) g(y) \cdots h(z) d x d y \cdots d z \\
\leq & K^{\lambda}\left(\frac{\bar{p}_{1}}{c}, \frac{\bar{p}_{2}}{c}, \ldots, \frac{\bar{p}_{r}}{c}\right) \\
& \left(\int w(x) d x\right)^{\alpha(1-\lambda)}\left(\int r(y) d y\right)^{\beta(1-\lambda)} \cdots\left(\int l(z) d z\right)^{\gamma(1-\lambda)} \\
& \left(\int f^{\frac{q_{1}}{c}}(x) d x\right)^{\frac{c}{q_{1}}}\left(\int g^{\frac{q_{2}}{c}}(y) d y\right)^{\frac{c}{q_{2}}} \cdots\left(\int h^{\frac{q_{r}}{c}}(z) d z\right)^{\frac{c}{q_{r}}},
\end{aligned}
$$

where $\bar{p}_{i}=\lambda\left(\lambda-1+\frac{1}{q_{i}}\right)^{-1}, i=1,2, \ldots, r$.

Proof. We need the following

Lemma. 4. Let $k(x, y, \ldots, z) \geq 0, m(x, y, \ldots, z) \geq 0, u_{1}>0, v_{1}>0, \ldots, w_{1}>$ $0, u_{2}>0, v_{2}>0, \ldots, w_{2}>0$ and suppose that for every nonnegative $f(x), g(y), \ldots$, $h(z)$ the following inequalities are valid

$$
\begin{aligned}
& \iint \cdots \int k(x, y, \ldots, z) f(x) g(y) \cdots h(z) d x d y \cdots d z \leq K_{1} \\
& \left(\int f(x)^{\frac{1}{u_{1}}} d x\right)^{u_{1}}\left(\int g(y)^{\frac{1}{v_{1}}} d y\right)^{v_{1}} \cdots\left(\int h(z)^{\frac{1}{w_{1}}} d z\right)^{w_{1}} \\
& \iint \cdots \int m(x, y, \ldots, z) f(x) g(y) \cdots h(z) d x d y \cdots d z \leq K_{2} \\
& \left(\int f(x)^{\frac{1}{u_{2}}} d x\right)^{u_{2}}\left(\int g(y)^{\frac{1}{v_{2}}} d y\right)^{v_{2}} \cdots\left(\int h(z)^{\frac{1}{w_{2}}} d z\right)^{w_{2}}
\end{aligned}
$$

Futher, let $k_{1}>0, k_{2}>0, k_{1}+k_{2}=1, u=k_{1} u_{1}+k_{2} u_{2}, v=k_{1} v_{1}+k_{2} v_{2}, \ldots, w=$ $k_{1} w_{1}+k_{2} w_{2}$. Then

$$
\begin{gathered}
\iint \cdots \int k^{k_{1}}(x, y, \ldots, z) m^{k_{2}}(x, y, \ldots, z) f(x) g(y) \cdots h(z) d x d y \cdots d z \leq K_{1}^{k_{1}} K_{2}^{k_{2}} \\
\left(\int f(x)^{\frac{1}{u}} d x\right)^{u}\left(\int g(y)^{\frac{1}{v}} d y\right)^{v} \cdots\left(\int h(z)^{\frac{1}{w}} d z\right)^{w} .
\end{gathered}
$$

Proof of Lemma 4. By Hölder inequality, since $k_{1}+k_{2}=1$, it follwos

$$
\begin{aligned}
& \iint \cdots \int k^{k_{1}}(x, y, \ldots, z) m^{k_{2}}(x, y, \ldots, z) f(x) g(y) \cdots h(z) d x d y \cdots d z \\
= & \iint \cdots \int\left(k f^{\frac{u_{1}}{u}} g^{\frac{v_{1}}{v}} \cdots h^{\frac{w_{1}}{w}}\right)^{k_{1}}\left(m f^{\frac{u_{2}}{{ }^{2}}} g^{\frac{v_{2}}{v}} \cdots h^{\frac{w_{2}}{w}}\right)^{k_{2}} d x d y \cdots d z
\end{aligned}
$$




$$
\begin{aligned}
\leq & \left(\iint \cdots \int k(x, y, \ldots, z) f^{\frac{u_{1}}{u}} g^{\frac{v_{1}}{v}} \cdots h^{\frac{w_{1}}{w}} d x d y \cdots d_{z}\right)^{k_{1}} \\
& \left(\iint \cdots \int m(x, y, \ldots, z) f^{\frac{u_{2}}{n}} g^{\frac{v_{2}}{v}} \cdots h^{\frac{w_{2}}{w}} d x d y \cdots d_{z}\right)^{k_{2}} .
\end{aligned}
$$

As consequences of (8) and (9), we have

$$
\begin{aligned}
& \iint \cdots \int k(x, y, \ldots, z) f^{\frac{u_{1}}{w}}(x) g^{\frac{v_{1}}{v}}(y) \cdots h^{\frac{w_{1}}{w}}(z) d x d y \cdots d z \\
\leq & K_{1}\left(\int f(x)^{\frac{1}{u}} d x\right)^{u_{1}}\left(\int g(y)^{\frac{1}{v}} d y\right)^{v_{1}} \cdots\left(\int h(z)^{\frac{1}{w}} d z\right)^{w_{1}} \\
& \iint \cdots \int m(x, y, \ldots, z) f^{\frac{u_{2}}{u}}(x) g^{\frac{v_{2}}{v}}(y) \cdots h^{\frac{w_{2}}{w}}(z) d x d y \cdots d z \\
\leq & K_{2}\left(\int f(x)^{\frac{1}{u}} d x\right)^{u_{2}}\left(\int g(y)^{\frac{1}{v}} d y\right)^{v_{2}} \cdots\left(\int h(z)^{\frac{1}{w}} d z\right)^{w_{2}} .
\end{aligned}
$$

Substituting these inequalities into (10), we obtain

$$
\begin{aligned}
& \iint \ldots \int k^{k_{1}}(x, y, \ldots, z) m^{k_{2}}(x, y, \ldots, z) f(x) g(y) \cdots h(z) d x d y \cdots d z \\
\leq & \left(K_{1}\left(\int f(x)^{\frac{1}{u}} d x\right)^{u_{1}}\left(\int g(y)^{\frac{1}{v}} d y\right)^{v_{1}} \cdots\left(\int h(z)^{\frac{1}{w}} d z\right)^{w_{1}}\right)^{k_{1}} \\
& \left(K_{2}\left(\int f(x)^{\frac{1}{u}} d x\right)^{u_{2}}\left(\int g(y)^{\frac{1}{v}} d y\right)^{v_{2}} \cdots\left(\int h(z)^{\frac{1}{w}} d z\right)^{w_{2}}\right)^{k_{2}} \\
= & K_{1}^{k_{1}} K_{2}^{k_{2}}\left(\int f^{\frac{1}{u}}(x) d x\right)^{k_{1} u_{1}+k_{2} u_{2}}\left(\int g^{\frac{1}{v}}(y) d y\right)^{k_{1} v_{1}+k_{2} v_{2}} \\
& \cdots\left(\int h^{\frac{1}{w}}(z) d z\right)^{k_{1} w_{1}+k_{2} w_{2}} \\
= & K_{1}^{k_{1}} K_{2}^{k_{2}}\left(\int f(x)^{\frac{1}{u}} d x\right)^{u}\left(\int g(y)^{\frac{1}{v}} d y\right)^{v} \cdots\left(\int h(z)^{\frac{1}{w}} d z\right)^{w} .
\end{aligned}
$$

Proof of Theorem 4. If we set $m(x, y, \ldots, z)=w^{\alpha}(x) r^{\beta}(y) \cdots l^{\gamma}(z), u_{1}=$ $\frac{c}{\overline{\bar{p}_{1}}}, v_{1}=\frac{c}{\overline{\bar{p}_{2}}}, \ldots, w_{1}=\frac{c}{\frac{\overline{p_{r}}}{q_{2}}}$ s.t. $\sum_{i=1}^{r} \frac{1}{\bar{p}_{i}}=1, u_{2}=v_{2}=\ldots=w_{2}=c, k_{1}=\lambda, u=\frac{c}{q_{1}}, v=$ $\frac{c}{q_{2}}, \ldots, w=\frac{c}{q_{r}}$. Since

$$
\left\{\begin{array}{l}
u=k_{1} u_{1}+k_{2} u_{2} \\
v=k_{1} v_{1}+k_{2} v_{2} \\
\vdots \\
w=k_{1} w_{1}+k_{2} w_{2}
\end{array}\right.
$$


we have

$$
\left\{\begin{array}{l}
\frac{1}{q_{1}}=\lambda \frac{1}{\overline{\bar{p}}_{1}}+k_{2} \\
\frac{1}{q_{2}}=\lambda \frac{1}{\bar{p}_{2}}+k_{2} \\
\vdots \\
\frac{1}{q_{r}}=\lambda \frac{1}{\bar{p}_{r}}+k_{2}
\end{array}\right.
$$

So that $k_{2}=1-\lambda$ and $\frac{1}{\bar{p}_{i}}=\frac{1}{\lambda}\left(\lambda-1+\frac{1}{q_{i}}\right), i=1,2, \ldots, r$

The conditions of Lemma 4 are fulfield, since we have $k_{2}>0, k_{1}+k_{2}=1$

Now to check $\bar{p}_{i}>1, i=1,2, \ldots, r$ where $\bar{p}_{i}=\lambda\left(\lambda-1+\frac{1}{q_{i}}\right)^{-1}$. We observe

$$
\begin{aligned}
\langle i\rangle \quad \lambda-1+\frac{1}{q_{i}} & =\frac{\sum_{j=1}^{r} \frac{1}{q_{j}^{\prime}}}{r-1}-1+\frac{1}{q_{i}} \\
& =\frac{\sum_{j=1}^{r} \frac{1}{q_{i}^{\prime}}-(r-1)+\frac{r-1}{q_{i}}}{r-1} \\
& =\frac{r-\sum_{j=1}^{r} \frac{1}{q_{j}}-r+1+\frac{r-1}{q_{i}}}{r-1} \\
& =\frac{1+\frac{r-1}{q_{i}}-\sum_{j=1}^{r} \frac{1}{q_{j}}}{r-1}>0
\end{aligned}
$$

and $\langle i i\rangle \quad \lambda-\left(\lambda-1+\frac{1}{q_{i}}\right)=1-\frac{1}{q_{i}}>0$.

It follows from $\langle i\rangle$ and $\langle i i\rangle$, that $\bar{p}_{i}>1, i=1,2, \ldots, r$.

Now we have:

$$
\begin{aligned}
& \iint \cdots \int k(x, y, \ldots, z) f(x) g(y) \cdots h(z) d x d y \cdots d z \\
\leq & K\left(\frac{\bar{p}_{1}}{c}, \frac{\bar{p}_{2}}{c}, \ldots, \frac{\bar{p}_{r}}{c}\right)\left(\int f^{\frac{\bar{p}_{1}}{c}}(x) d x\right)^{\frac{c}{\bar{p}_{1}}}\left(\int g^{\frac{\bar{p}_{2}}{c}}(y) d y\right)^{\frac{c}{\bar{p}_{2}}} \cdots\left(\int h^{\frac{\bar{p}_{r}}{c}}(z) d z\right)^{\frac{c}{\bar{p}_{r}}} \\
= & K_{1}\left(\int f(x)^{\frac{1}{u_{1}}} d x\right)^{u_{1}}\left(\int g(y)^{\frac{1}{v_{1}}} d y\right)^{v_{1}}\left(\int h(z)^{\frac{1}{w_{1}}} d z\right)^{w_{1}} .
\end{aligned}
$$

where $K_{1}=K\left(\frac{\bar{p}_{1}}{c}, \frac{\bar{p}_{2}}{c}, \ldots, \frac{\bar{p}_{r}}{c}\right)$

Futhermore, Since $\alpha+c=1, \beta+c=1, \ldots, \gamma+c=1$, it follwos from Hölder inequality 
that:

$$
\begin{aligned}
& \iint \cdots \int w^{\alpha}(x) r^{\beta}(y) \cdots l^{\gamma}(z) f(x) g(y) \cdots h(z) d x d y \cdots d z \\
\leq & \left(\int w^{\alpha}(x)\left(f^{\frac{1}{c}}(x)\right)^{c} d x\right)\left(\int r^{\beta}(y)\left(g^{\frac{1}{c}}(y)\right)^{c} d y\right) \cdots\left(\int l^{\gamma}(z)\left(h^{\frac{1}{c}}(z)\right)^{c} d z\right) \\
\leq & \left(\int w(x) d x\right)^{\alpha}\left(\int f^{\frac{1}{c}}(x) d x\right)^{c}\left(\int r(y) d y\right)^{\beta}\left(\int g^{\frac{1}{c}}(y) d y\right)^{c} \\
& \cdots\left(\int l(z) d z\right)^{\gamma}\left(\int h^{\frac{1}{c}}(z) d z\right)^{c} \\
= & K_{2}\left(\int f(x)^{\frac{1}{u_{2}}} d x\right)^{u_{2}}\left(\int g(y)^{\frac{1}{v_{2}}} d y\right)^{v_{2}} \cdots\left(\int h(z)^{\frac{1}{w_{2}}} d z\right)^{w_{2}},
\end{aligned}
$$

where $K_{2}=\left(\int w(x) d x\right)^{\alpha}\left(\int r(y) d y\right)^{\beta} \cdots\left(\int l(z) d z\right)^{\gamma}$.

It follows from Lemma 4 that

$$
\begin{aligned}
& \iint \cdots \int k^{\lambda}(x, y, \ldots, z)\left(w^{\alpha}(x) r^{\beta}(y) \cdots l^{\gamma}(z)\right)^{1-\lambda} f(x) g(y) \cdots h(z) d x d y \cdots d z \\
\leq & K^{\lambda}\left(\frac{\bar{p}_{1}}{c}, \frac{\bar{p}_{2}}{c}, \ldots \frac{\bar{p}_{r}}{c}\right)\left(\int w(x) d x\right)^{\alpha(1-\lambda)}\left(\int r(y) d y\right)^{\beta(1-\lambda)} \cdots\left(\int l(z) d z\right)^{\gamma(1-\lambda)} \\
& \left(\int f^{\frac{q_{1}}{c}}(x) d x\right)^{\frac{c}{q_{1}}}\left(\int g^{\frac{q_{2}}{c}}(y) d y\right)^{\frac{c}{q_{2}}} \cdots\left(\int h^{\frac{q_{r}}{c}}(z) d z\right)^{\frac{c}{q_{r}}}
\end{aligned}
$$

where $\bar{p}_{i}=\lambda\left(\lambda-1+\frac{1}{q_{i}}\right)^{-1}, i=1,2, \ldots, r$.

This completes the proof of Theorem 4 .

\section{References}

[1] F. F. Bonsall, "Inequalities with non-conjugate parameters," Quart. J. Math. Oxford Ser., (2) 2 (1951), 135-150.

[2] G. H. Hardy, J. E. Littlewood, and G. Pólya, Inequalities, Cambridge, 1934, and 1952.

[3] D. S. Mitrinorić and J. E. Pečarič, "A note on an inequality with non-conjugate parameters , Sitzungsberichte der mathem", -naturw. KL. Abt., II, 199. Band, 8. -10. Heft (1990), 155-160.

[4] V. Levin, "On the two-parameter extension and analogue of Hilbert's inequality," London Math. Soc., 11 (1936), 119-124.

[5] S. B. Stečkin, "On positive bilinear forms (Russian)," Dokl. Akad. Nauk., SSSR 65 (1949), Nr.1, $17-20$.

Department of Mathematics, Tamkang University, Tamsui, Taiwan 25137. 